\title{
Sensitivity of Various Evaluating Modalities for Predicting a Pathologic Complete Response After Preoperative Chemoradiation Therapy for Locally Advanced Rectal Cancer
}

\author{
Sungwoo Jung ${ }^{1, \star}$, Anuj Parajuli²,* Chang Sik Yu ${ }^{3}$, Seong Ho Park ${ }^{4}$, Jong Seok Lee ${ }^{4}$, Ah Young Kim \\ Jong Lyul Lee 3 , Chan Wook Kim³, Yong Sik Yoon ${ }^{3}$, In Ja Park ${ }^{3}$, Seok-Byung Lim ${ }^{3}$, Jin Cheon Kim³ \\ ${ }^{1}$ Division of Acute Care Surgery, Department of Surgery, Asan Medical Center, University of Ulsan College of Medicine, Seoul, Korea; \\ ${ }^{2}$ Department of Surgery, Kathmandu Medical College Teaching Hospital, Kathmandu, Nepal; ${ }^{3}$ Division of Colon and Rectal Surgery, \\ Department of Surgery, Asan Medical Center, University of Ulsan College of Medicine, Seoul; ${ }^{4}$ Department of Radiology and the Research \\ Institute of Radiology, Asan Medical Center, University of Ulsan College of Medicine, Seoul, Korea
}

Purpose: We investigated the sensitivity of various evaluating modalities in predicting a pathologic complete response (pCR) after preoperative chemoradiation therapy (PCRT) for locally advanced rectal cancer (LARC).

Methods: From a population of 2,247 LARC patients who underwent PCRT followed by surgery at Asan Medical Center, Seoul, Korea from January 2007 to June 2016, we retrospectively analyzed 313 patients (14.1\%) who showed a pCR after surgery. Transrectal ultrasound (TRUS), high-resolution magnetic resonance imaging (MRI), abdominopelvic computed tomography (AP-CT), and endoscopy were performed within 2 weeks prior to surgery.

Results: Of the 313 patients analyzed, $256(81.8 \%)$ had a pCR after radical surgery and 57 (18.2\%) showed total regression after local excision. Preoperative TRUS, MRI, and AP-CT were performed in 283, 305, and 139 patients, respectively. Among these 3 groups, a prediction of a pCR of the primary tumor was made in 41 (14.5\%), 51 (16.7\%), and 27 patients (19.4\%), respectively, before surgery. A prediction of a clinical N0 stage was made in 204 patients (88.3\%) using TRUS, 130 (52.2\%) using MRI, and 78 (65.5\%) using AP-CT. Of the 211 patients who underwent endoscopy, 87 (41.2\%) had a mention of clinical CR in their records. A prediction of a pathologic CR was made for 124 patients (39.6\%) through at least one diagnostic modality.

Conclusion: The various evaluation methods for predicting a pCR after PCRT show a predictive sensitivity of $0.15-0.41$ for primary tumors and $0.52-0.88$ for lymph nodes. Endoscopy is a relatively superior modality for predicting the pCR of the primary tumor of LARC patients.

Keywords: Preoperative chemoradiotherapy; Complete regression; Response prediction

Received: November 30, 2018 - Accepted: January 7, 2019

Correspondence to: Chang Sik Yu, M.D.

Division of Colon and Rectal Surgery, Department of Surgery, Asan Medical Center, University of Ulsan College of Medicine, 88 Olympic-ro 43-gil, Songpa-gu, Seoul 05505, Korea

Tel: +82-2-3010-3494; Fax: +82-2-3010-6701, E-mail: csyu@amc.seoul.kr ORCID code: https://orcid.org/0000-0001-9401-9981

*Sunwoo Jung and Anuj Parajuli contributed equally as co-first authors.

(c) 2019 The Korean Society of Coloproctology

This is an open-access article distributed under the terms of the Creative Commons Attribution NonCommercial License (http://creativecommons.org/licenses/by-nc/4.0) which permits unrestricted noncommercial use, distribution, and reproduction in any medium, provided the original work is properly cited.

\section{INTRODUCTION}

Preoperative chemoradiation therapy (PCRT) followed by total mesorectal excision (TME) is the standard treatment regimen for locally advanced rectal cancer (LARC, T3-4 and/or N+) [1]. Approximately $8 \%-24 \%$ of patients show a pathologic complete response (pCR) to this treatment with improved long-term oncologic outcomes and local control $[2,3]$. On the basis of these promising results, more conservative alternatives to TME, including "wait-and-watch" and "local excision," have been used in patients who are expected to achieve pCR after PCRT with good 
outcomes $[2,4,5]$.

Notably, accurate restaging after PCRT is indispensable when adopting a conservative therapeutic approach in LARC patients in whom a pCR, i.e., a clinical complete response (cCR), is anticipated. Various imaging modalities have been used to accurately predict a pCR prior to surgery including magnetic resonance imaging (MRI) [6-11], abdominopelvic computed tomography (APCT) $[12,13]$, transrectal ultrasound (TRUS) [14-16], and endoscopy (colonoscopy and sigmoidoscopy) $[17,18]$. However, most studies on the pCR-prediction accuracy of each of these tests have reported poor predictive power.

In this study, we investigated the pCR-prediction sensitivity of various restaging modalities in a select cohort of LARC patients with a final confirmation of pCR after PCRT followed by surgery.

\section{METHODS}

\section{Patients and study design}

A total of 2,247 patients underwent PCRT followed by surgery for biopsy-proven LARC at Asan Medical Center, Seoul, Korea between January 2007 and June 2016. We enrolled 313 patients from this population $(14.1 \%)$ for a retrospective evaluation because they either showed pCR after radical surgery or total regression after local excision. The patients in our study with clinical T3-4 and/or N+ mid-to-lower rectal cancer underwent PCRT. Most of these subjects also underwent TRUS, AP-CT, chest CT, high-resolution rectal MRI, endoscopy (colonoscopy or sigmoidoscopy in patients with tumor obstruction) to confirm the clinical stage and location of the tumor. This study was approved by the Institutional Review Board (IRB) of Asan Medical Center (registration number: S2017-2141-0001), and the requirement for informed consent was waived by the IRB.

\section{Preoperative chemoradiation therapy}

Preoperative radiotherapy consisted of 25 fractions at a dosage of 45-50 Gy administered to the entire pelvis followed by a 5.4-Gy boost to the primary tumor administered in 3 fractions. Chemotherapy was delivered in 2 cycles with either an intravenous bolus of 5-fluorouracil $\left(375 \mathrm{mg} / \mathrm{m}^{2} /\right.$ day $)$ and leucovorin $\left(20 \mathrm{mg} / \mathrm{m}^{2} /\right.$ day) over 3 days during the first and fifth weeks of radiation therapy or with oral capecitabine $\left(1,650 \mathrm{mg} / \mathrm{m}^{2} /\right.$ day $)$ twice-daily during the period of radiation therapy.

\section{Restaging and surgery}

Restaging using TRUS, high-resolution rectal MRI, AP-CT, or endoscopy (colonoscopy or sigmoidoscopy) was performed within 2 weeks prior to surgery. All TRUSs were performed by colorectal surgeons. Experienced gastrointestinal (GI) radiologists interpreted all MRI and AP-CT findings. According to the judgment of the surgeon, the colonoscopy or sigmoidoscopy was performed by either a colorectal surgeon or a GI endoscopist to identify remnant tumors or cCR. Patients with $\mathrm{cCR}$ were defined as those with the possibility of a $\mathrm{pCR}$ as determined by the physician who performed or interpreted each test. The operations were planned within 6-8 weeks of the completion of PCRT. The surgeon's decision to perform either a radical resection according to the principles of tumor-specific mesorectal excision or a local excision was based on the restaging result and the patient's general status. The patients who were classified as magnetic resonance tumor regression grade (mrTRG) 1 (i.e., radiological complete remission) or 2 (i.e., dense fibrosis without obvious tumor signal) after PCRT underwent local excision. The pathologic stage was recorded in accordance with the seventh edition of the TNM classification of the American Joint Committee on Cancer [19]. Pathologic CR was confirmed in the resected specimens using the tumor regression grade system described previously by Dworak et al. [20].

\section{Statistical analysis}

Post-PCRT clinical $\mathrm{T}$ and $\mathrm{N}$ stages were determined using pelvic TRUS, AP-CT, MRI, and endoscopy. The sensitivity of predicting ypT0 and ypN0 was calculated for each imaging modality individually. The overall predictive sensitivity was also measured for patients expected to achieve a pCR by collecting the best results from all the imaging tests.

\section{RESULTS}

\section{Demographic characteristics and surgical procedures}

Among the 313 patients analyzed in the current study, there were 189 males (60.4\%) and 124 females (39.6\%). Of the study population, 256 patients (81.8\%) showed pCR after TME and 57 (18.2\%) showed total regression after local excision. At the time of initial diagnosis, the clinical T3 stage was the most frequent with 248 subjects (79.2\%) followed by clinical $\mathrm{N}+$ with 261 subjects (83.4\%). The median interval between the last date of radiotherapy and the date of the surgery was 7 weeks (Table 1).

\section{Prediction sensitivity for ypT0 and ypN0 staging}

Post-PCRT restaging was performed using TRUS (283 of 313 , 90.4\%), AP-CT (139 of 313, 44.4\%), and MRI (305 of 313, 97.4\%) (Fig. 1). Using TRUS, 41 (14.5\%, sensitivity 0.15 ) of our study patients were predicted to have a ypT0-stage lesion and 204 (88.3\%, sensitivity 0.88 ) to have a ypN0-stage tumor. For $\mathrm{T}$ and $\mathrm{N}$ staging with MRI, 51 (16.7\%, sensitivity 0.17$)$ patients were staged as T0 and $130(52.2 \%$, sensitivity 0.52$)$ as N0. Comparable results were obtained using AP-CT in which $27(19.4 \%$, sensitivity 0.19$)$ patients were staged as T0 and 78 (65.5\%, sensitivity 0.66$)$ as N0s (Tables 2 and 3). The concordance between MRI and other restaging methods in terms of ycT stage and ycN stage is shown in Table 4.

Preoperative re-endoscopy was performed in 211 of our study patients (67.4\%) with the possibility of CR in 87 cases $(41.2 \%$, sensitivity 0.41 ) due to the detection of only a shallow ulcer or 
Table 1. Clinicodemographic characteristics of patients with pathologic complete response

\begin{tabular}{|c|c|}
\hline Variable & Value \\
\hline Age (yr) & $56(26-83)$ \\
\hline \multicolumn{2}{|l|}{ Sex } \\
\hline Male & $189(60.4)$ \\
\hline Female & $124(39.6)$ \\
\hline \multicolumn{2}{|l|}{ Operation type } \\
\hline Radical resection & $256(81.8)$ \\
\hline uLAR & $206(65.8)$ \\
\hline APR & $50(16.0)$ \\
\hline Local excision & 57 (18.2) \\
\hline TAE & 39 (12.5) \\
\hline TAMIS & $18(5.8)$ \\
\hline \multicolumn{2}{|l|}{ Approach } \\
\hline Open & $183(58.5)$ \\
\hline Laparoscopic assisted & $25(8.0)$ \\
\hline Robot assisted & 48 (15.3) \\
\hline Local excision & 57 (18.2) \\
\hline \multicolumn{2}{|l|}{ Interval (wk) } \\
\hline PCRT to surgery & $7(4-41)$ \\
\hline PCRT to restaging & $5.4(0.7-7)$ \\
\hline Restaging to surgery & $1.4(0.1-12.4)$ \\
\hline Distance from anal verge $(\mathrm{cm})$ & $4(0-10)$ \\
\hline \multicolumn{2}{|l|}{ Clinical T stage } \\
\hline $\mathrm{T} 2$ & $34(10.9)$ \\
\hline T3 & $248(79.2)$ \\
\hline T4 & $31(9.9)$ \\
\hline \multicolumn{2}{|l|}{ Clinical N stage } \\
\hline NO & $52(16.6)$ \\
\hline $\mathrm{N}+$ & 261 (83.4) \\
\hline
\end{tabular}

Values are presented as median (range) or number (\%).

ULAR, ultralow anterior resection; APR, abdominoperineal resection; TAE, transanal excision; TAMIS, transanal minimally invasive surgery; PCRT, preoperative chemoradiation therapy.

scar or a polypoid lesion (Table 5, Fig. 2).

\section{Overall sensitivity}

The predictive sensitivity was 0.4 for all of the tests combined, and 124 patients (39.6\%) in our study cohort were predicted to have pCR.

\section{DISCUSSION}

The sensitivity of the evaluation methods tested in our current
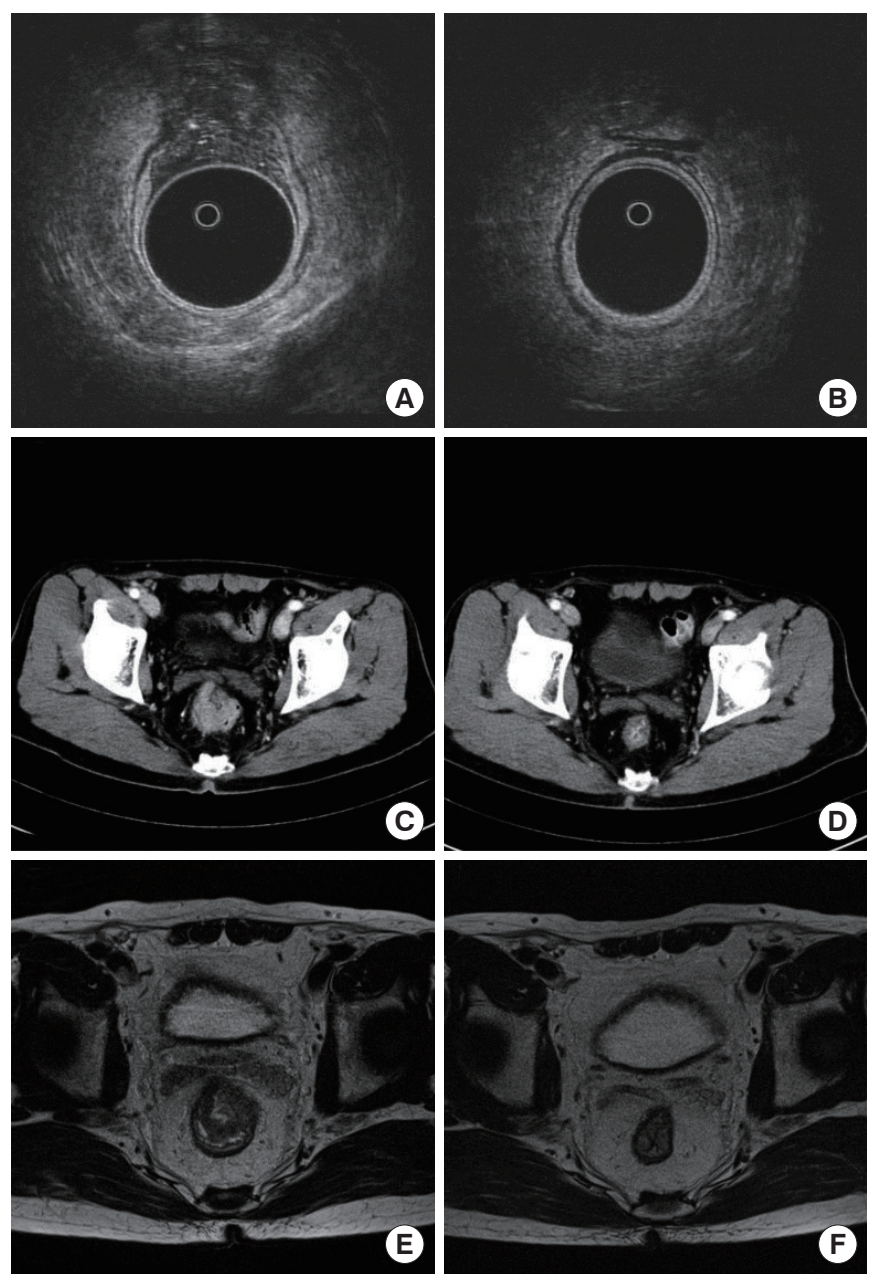

Fig. 1. Evaluating images of a 66-year-old man diagnosed with clinical T3N+ rectal cancer at initial evaluation. (A) TRUS before PCRT: the hypoechoic mass extended over the muscularis propria into the perirectal fat from the 10-o'clock position to the 3-o'clock position. (B) TRUS after PCRT: the previous hypoechoic mass is gone. (C) AP-CT before PCRT: an irregularly enhanced mass in the anterior rectal wall. (D) AP-CT after PCRT: the previous rectal mass is gone. (E) T2W MRI before PCRT: T3 rectal cancer with heterogeneous signal intensity from the 10-o'clock position to the 3-o'clock position. (F) T2W MRI after PCRT: the previous rectal tumor is gone. TRUS, transrectal ultrasound; PCRT, preoperative chemoradiation therapy; AP-CT, abdominopelvic computed tomography; T2W MRI, T2weighted magnetic resonance imaging.

study to accurately predict a pCR after PCRT in patients with LARC over a 10 -year period was $0.15-0.41$ for primary tumors and $0.52-0.88$ for lymph nodes (LNs). Endoscopy had the highest pCR-prediction sensitivity (0.41) among the conventional examination tools that were tested. It is noteworthy that almost $60 \%$ of the cases of pCR after treatment in this study were not accurately predicted by any of the conventional restaging modalities. Al- 
though the current study was retrospective, a large number of pCR patients were comprehensively analyzed, which included an assessment of all conventional tests currently used for restaging after PCRT.

The sensitivity of MRI for predicting pCR was 0.17 for primary tumors and 0.52 for LNs. These results were comparable to those of a recent meta-analysis of the performance of MRI for restaging that reported a mean pCR-predictive sensitivity for primary tumors of 0.19 and a sensitivity of the nodal staging per patient of 0.77 [6]. Although some studies have tested the mrTRG and diffusion-weighted MRI in predicting PCRT response and oncologic outcomes [7,8], conventional MRI has not previously been found to accurately predict the response to PCRT [9-11]. Another metaanalysis reported an average accuracy of 52\% when using restaging MRI to predict the T stage and that $73 \%$ of ypT0 tumors were overstaged with this method. That meta-analysis concluded that restaging MRI had only played a role in excluding CRM involvement [21]. Furthermore, van den Broek et al. [22] reported a 0\% positive predictive value for $\mathrm{CCR}$ in 46 patients and that no

Table 2. Clinical T-staging results with various imaging modalities

\begin{tabular}{lccc}
\hline cT stage & TRUS & AP-CT & MRI \\
\hline T0 & $41(14.5)$ & $27(19.4)$ & $51(16.7)$ \\
T1 & $12(4.2)$ & $2(1.4)$ & $9(3.0)$ \\
T2 & $42(14.8)$ & $54(38.8)$ & $106(34.8)$ \\
T3 & $182(64.3)$ & $47(33.8)$ & $126(41.3)$ \\
T4 & $6(2.1)$ & $9(6.5)$ & $13(4.3)$ \\
Total & $283(100)$ & $139(100)$ & $305(100)$ \\
\hline
\end{tabular}

Values are presented as number (\%).

TRUS, transrectal ultrasound; AP-CT, abdominopelvic computed tomography; MRI, magnetic resonance imaging.

Table 3. Clinical $\mathrm{N}$ staging results with various imaging modalities

\begin{tabular}{lccc}
\hline cN stage & TRUS & AP-CT & MRI \\
\hline No & $204(88.3)$ & $78(65.5)$ & $130(52.2)$ \\
$\mathrm{N}+$ & $27(11.7)$ & $41(34.5)$ & $119(47.8)$ \\
Total & $231(100)$ & $139(100)$ & $249(100)$ \\
\hline
\end{tabular}

Values are presented as number (\%).

TRUS, transrectal ultrasound; AP-CT, abdominopelvic computed tomography; MRI, magnetic resonance imaging. changes were made to the surgical planning by their multidisciplinary team as a result of restaging MRI.

The remaining LN size is an important criterion for determining whether LN metastases remain after PCRT. Pomerri et al. [23] reported that the NPV value for a negative LN status was $97 \%$ $100 \%$ when the LN size was reduced by more than $70 \%$ as determined using MRI after PCRT. However, it should be noted that
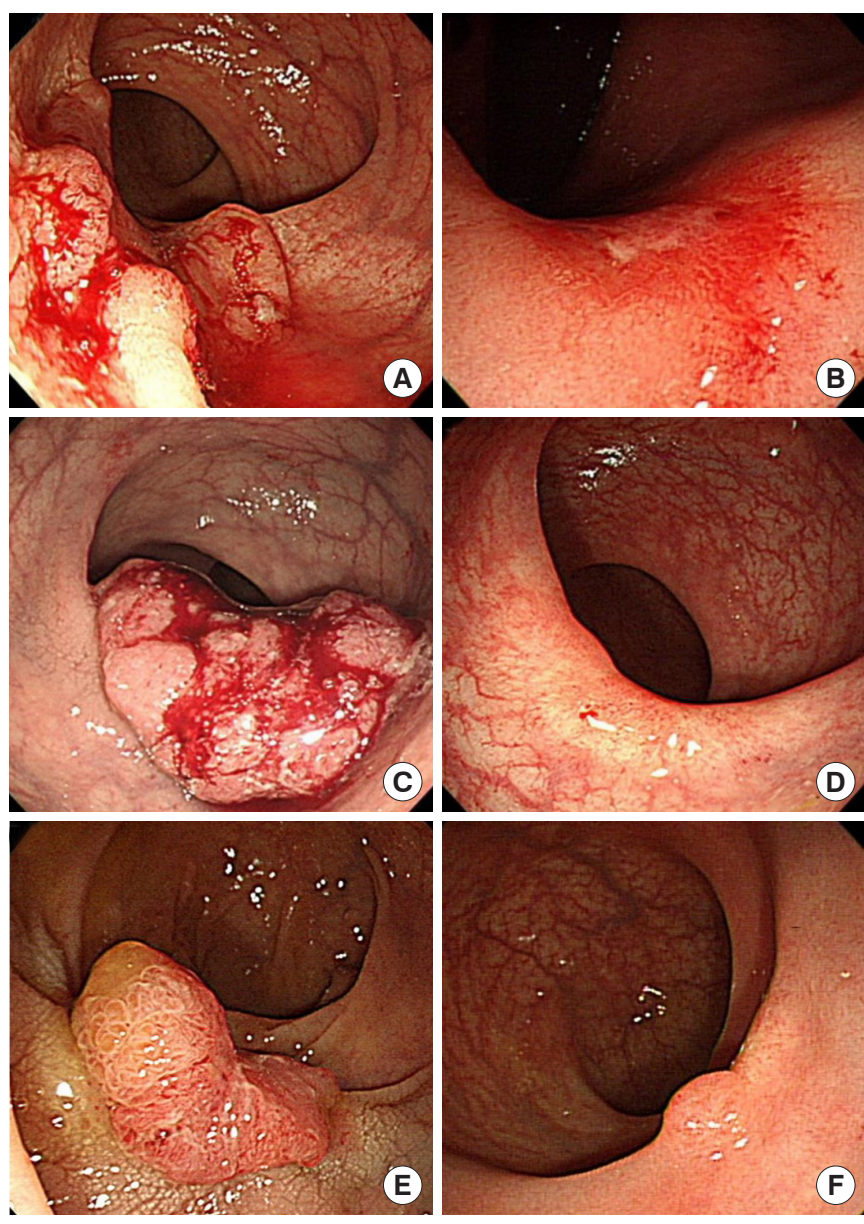

Fig. 2. (A, C, E) Endoscopic findings of patients with locally advanced rectal cancer before preoperative chemoradiation therapy (PCRT). (B, D, F) Endoscopic findings described as clinical complete response after PCRT. (C) Hyperemic mucosal change with a shallow ulcer. (D) Fibrotic scar. (F) A polypoid lesion with fibrotic scar.

Table 4. Concordance between MRI and other restaging methods in ycT and ycN stage

\begin{tabular}{lccrr}
\hline Variable & ycT0 & All ycT stages & \multicolumn{1}{c}{ ycN0 } & All ycN stages \\
\hline MRI vs. TRUS & $21 / 48(43.8)$ & $144 / 278(51.8)$ & $114 / 118(96.6)$ & $136 / 227(59.9)$ \\
MRI vs. AP-CT & $18 / 23(78.3)$ & $107 / 135(79.3)$ & $60 / 64(93.8)$ & $95 / 115(82.6)$ \\
MRI vs. endoscopy & $29 / 38(76.3)$ & $140 / 206(68.0)$ & - & - \\
\hline
\end{tabular}

Values are presented as number (\%).

MRI, magnetic resonance imaging; TRUS, transrectal ultrasound; AP-CT, abdominopelvic computed tomography. 
Table 5. Summary of endoscopic findings

\begin{tabular}{lc}
\hline Endoscopic findings & Value \\
\hline Clinical complete response & $87(41.2)$ \\
Scar & $54(25.6)$ \\
Ulcer & $29(13.7)$ \\
Polypoid & $4(1.9)$ \\
Remnant tumors & $124(58.8)$ \\
Ulceroinfiltrative & $73(34.6)$ \\
Ulcerofungating & $51(24.2)$ \\
Total & $211(100)$ \\
\hline
\end{tabular}

Values are presented as number (\%).

even if several LNs are found to be reduced in size after PCRT, radiologists are likely to be hesitant to interpret them as negative if they had been assessed as metastases on MRI before PCRT because a marked number of metastatic LNs of below $3 \mathrm{~mm}$ have been reported $[24,25]$. This is likely the cause of overstaging of the LN status in patients showing a pCR.

AP-CT is performed in less than half of patients who have undergone PCRT because there is a belief among some surgeons that there is no additional benefit to performing another test beyond MRI. However, the current analysis demonstrated that the pCR rate with AP-CT was higher for both primary tumors and LNs compared with MRI. Because of the greater soft-tissue contrast and resolution of MRI compared to CT, it is to be expected that MRI will more frequently detect residual abnormalities after CRT, even if they actually represent complete radiation therapy changes without residual tumor cells [26]. This could largely explain the lower sensitivity of MRI for predicting a pCR compared with CT. However, several studies have shown that AP-CT also cannot accurately predict $\mathrm{PCR}[12,13]$.

The sensitivity of TRUS for predicting pCR in the current study was low for primary tumors $(0.15)$ but relatively high for the $\mathrm{N}$ stage $(0.88)$. In patients with an advanced primary tumor prior to PCRT, correctly staging a ypT0 tumor may be problematic with TRUS because regressed primary tumors can appear to be remnant lesions when using this method. Conversely, the N0 prediction rate is high using TRUS. Also, extramesorectal LNs cannot be accurately evaluated with TRUS because their location is beyond the detectable range of this method leading to a higher negative result rate [14]. Consequently, some previous studies have suggested that TRUS may be a useful method of evaluation in cases of node-negative rectal cancer but is an inaccurate modality for predicting the PCRT response for tumors in the rectal wall [15, $16]$.

Two hundred eleven (67.4\%) of our current study patients were re-evaluated with endoscopy after PCRT. The results of these tests indicated pCR-possible findings in 87 cases (41.2\%), which was relatively higher than any of the other evaluating modalities we tested. Maas et al. [27] previously included "no residual tumor at endoscopy or only a small residual erythematous ulcer or scar, negative biopsies from the scar, ulcer, or former tumor location" as cCR criteria for "wait-and-watch". In addition, endoscopic findings suggestive of $\mathrm{cCR}$ have previously been presented as "no palpable ulcer, nodule, mass, whitening of the mucosa, and telangiectasia" [17]. Kawai et al. [18] previously reported pCR-prediction sensitivities of $0.65-0.87$ through their endoscopic assessment criteria and determined that a flattened marginal swelling (odds ratio [OR], 3.39) and cancer-negative biopsy (OR, 4.61) were significant independent predictors of pCR. Endoscopy alone cannot be used for tumor staging but can play an important role in judging pCR and should be regarded as a useful re-evaluation tool after PCRT along with other imaging modalities. Notably, however, although many previous reports including the aforementioned studies have described criteria for $\mathrm{CCR}$ and $\mathrm{pCR}$ prediction, no concrete and objective guidelines have been presented to date that has gained global acceptance. Furthermore, endoscopic findings in rectal cancer patients can differ depending on the diagnostic judgment of the endoscopist, and clear standardized criteria are therefore also needed for this modality.

There were some notable limitations to our current investigation. Although a relatively large number of patients with $\mathrm{pCR}$ were analyzed, only the sensitivity of the tests was determined. Specificity, positive predictive value, and negative predictive value (NPV) are required as well as sensitivity to measure the diagnostic accuracy of the evaluating modalities in predicting pCR before surgery. However, this investigation focused on patients who were confirmed as pCR and how many patients among them were predicted ypCR using these conventional modalities. Further studies will be conducted to determine additional accuracy parameters.

A second limitation was that our analysis was based on clinical readings rather than a standardized central review of patient examinations. Since various radiologists, endoscopists, and colorectal surgeons performed these examinations and were involved in the restaging evaluations, there may have been inconsistencies in the quality of the observations and interpretations and in the criteria used to make them. Furthermore, unlike the interpretation of pre-CRT imaging examinations [28], standardization in the interpretation of post-CRT imaging examinations is still underway. These issues, in combination with the fact that the complete response of rectal cancer to CRT was not a well-established concept during much of the study period (and is in fact still evolving), suggest that indeterminate findings during follow-up were not likely to be deemed as PCR by clinicians and surgeons. It would be interesting to determine how the use of more standardized recent approaches, such as mrTRG $[7,29]$, might affect this situation. However, we believe that our present results accurately reflect the current predictive sensitivity for pCR in real-world clinical practice. In addition to the limitations in interpreting MRIs, the lack of well-structured criteria for endoscopy regarding cCR is another limitation. Since the reported endoscopic results are likely to depend on the subjective judgment of endoscopists, a stan- 
dardized reporting format should be established for accurate prediction of pCR. A third limitation is that restaging 4 to 6 weeks after PCRT might be too soon to assess pCR due to post-CRT edema and inflammatory changes [30]. Also, the median of 1.4 weeks between restaging and surgery may have decreased the predictive sensitivity. Another limitation was that we did not analyze specificity, the positive and NPVs, or accuracy of the prediction. We attempted to identify how accurately pCR had been predicted using only LARC patients who were eventually confirmed to have pCR but found a sensitivity of only 0.4 .

Despite the availability of various restaging modalities for predicting pCR after PCRT, the predictive sensitivity is only approximately 0.4 for primary tumors and $0.52-0.88$ for LNs. Endoscopy does show relatively superior performance for $\mathrm{pCR}$ prediction in the case of primary tumors and therefore should be used to evaluate all patients after PCRT. In the absence of a robust method to accurately predict pCR in LARC patients, comprehensive evaluations by experienced clinicians using conventional methods, including endoscopy, could increase the pCR-predictive power.

\section{CONFLICT OF INTEREST}

No potential conflict of interest relevant to this article was reported.

\section{REFERENCES}

1. Benson AB 3rd, Venook AP, Bekaii-Saab T, Chan E, Chen YJ, Cooper HS, et al. Rectal cancer, version 2.2015. J Natl Compr Canc Netw 2015;13:719-28.

2. García-Aguilar J, Hernandez de Anda E, Sirivongs P, Lee SH, Madoff RD, Rothenberger DA. A pathologic complete response to preoperative chemoradiation is associated with lower local recurrence and improved survival in rectal cancer patients treated by mesorectal excision. Dis Colon Rectum 2003;46:298-304.

3. Maas M, Nelemans PJ, Valentini V, Das P, Rödel C, Kuo LJ, et al. Long-term outcome in patients with a pathological complete response after chemoradiation for rectal cancer: a pooled analysis of individual patient data. Lancet Oncol 2010;11:835-44.

4. Yu CS, Yun HR, Shin EJ, Lee KY, Kim NK, Lim SB, et al. Local excision after neoadjuvant chemoradiation therapy in advanced rectal cancer: a national multicenter analysis. Am J Surg 2013;206: 482-7.

5. Habr-Gama A, Sabbaga J, Gama-Rodrigues J, São Julião GP, Proscurshim I, Bailão Aguilar P, et al. Watch and wait approach following extended neoadjuvant chemoradiation for distal rectal cancer: are we getting closer to anal cancer management? Dis Colon Rectum 2013;56:1109-17.

6. van der Paardt MP, Zagers MB, Beets-Tan RG, Stoker J, Bipat S. Patients who undergo preoperative chemoradiotherapy for locally advanced rectal cancer restaged by using diagnostic MR imaging: a systematic review and meta-analysis. Radiology 2013;269:101-
12.

7. Patel UB, Taylor F, Blomqvist L, George C, Evans H, Tekkis P, et al. Magnetic resonance imaging-detected tumor response for locally advanced rectal cancer predicts survival outcomes: MERCURY experience. J Clin Oncol 2011;29:3753-60.

8. Lambregts DM, Vandecaveye V, Barbaro B, Bakers FC, Lambrecht M, Maas M, et al. Diffusion-weighted MRI for selection of complete responders after chemoradiation for locally advanced rectal cancer: a multicenter study. Ann Surg Oncol 2011;18:2224-31.

9. Hötker AM, Garcia-Aguilar J, Gollub MJ. Multiparametric MRI of rectal cancer in the assessment of response to therapy: a systematic review. Dis Colon Rectum 2014;57:790-9.

10. Curvo-Semedo L, Lambregts DM, Maas M, Thywissen T, Mehsen RT, Lammering G, et al. Rectal cancer: assessment of complete response to preoperative combined radiation therapy with chemotherapy--conventional MR volumetry versus diffusionweighted MR imaging. Radiology 2011;260:734-43.

11. Ryan JE, Warrier SK, Lynch AC, Ramsay RG, Phillips WA, Heriot AG. Predicting pathological complete response to neoadjuvant chemoradiotherapy in locally advanced rectal cancer: a systematic review. Colorectal Dis 2016;18:234-46.

12. Guillem JG, Ruby JA, Leibold T, Akhurst TJ, Yeung HW, Gollub MJ, et al. Neither FDG-PET Nor CT can distinguish between a pathological complete response and an incomplete response after neoadjuvant chemoradiation in locally advanced rectal cancer: a prospective study. Ann Surg 2013;258:289-95.

13. Maretto I, Pomerri F, Pucciarelli S, Mescoli C, Belluco E, Burzi S, et al. The potential of restaging in the prediction of pathologic response after preoperative chemoradiotherapy for rectal cancer. Ann Surg Oncol 2007;14:455-61.

14. Akasu T, Sugihara K, Moriya Y, Fujita S. Limitations and pitfalls of transrectal ultrasonography for staging of rectal cancer. Dis Colon Rectum 1997;40(10 Suppl):S10-S15.

15. Rau B, Hünerbein M, Barth C, Wust P, Haensch W, Riess H, et al. Accuracy of endorectal ultrasound after preoperative radiochemotherapy in locally advanced rectal cancer. Surg Endosc 1999; 13:980-4.

16. Huh JW, Park YA, Jung EJ, Lee KY, Sohn SK. Accuracy of endorectal ultrasonography and computed tomography for restaging rectal cancer after preoperative chemoradiation. J Am Coll Surg 2008;207:7-12.

17. Habr-Gama A, Perez RO, Wynn G, Marks J, Kessler H, Gama-Rodrigues J. Complete clinical response after neoadjuvant chemoradiation therapy for distal rectal cancer: characterization of clinical and endoscopic findings for standardization. Dis Colon Rectum 2010;53:1692-8.

18. Kawai K, Ishihara S, Nozawa H, Hata K, Kiyomatsu T, Morikawa T, et al. Prediction of pathological complete response using endoscopic findings and outcomes of patients who underwent watchful waiting after chemoradiotherapy for rectal cancer. Dis Colon Rectum 2017;60:368-75.

19. Edge SB, Compton CC. The American Joint Committee on Can- 
cer: the 7th edition of the AJCC cancer staging manual and the future of TNM. Ann Surg Oncol 2010;17:1471-4.

20. Dworak O, Keilholz L, Hoffmann A. Pathological features of rectal cancer after preoperative radiochemotherapy. Int J Colorectal Dis 1997;12:19-23.

21. Memon S, Lynch AC, Bressel M, Wise AG, Heriot AG. Systematic review and meta-analysis of the accuracy of MRI and endorectal ultrasound in the restaging and response assessment of rectal cancer following neoadjuvant therapy. Colorectal Dis 2015;17: 748-61.

22. van den Broek JJ, van der Wolf FS, Lahaye MJ, Heijnen LA, Meischl C, Heitbrink MA, et al. Accuracy of MRI in restaging locally advanced rectal cancer after preoperative chemoradiation. Dis Colon Rectum 2017;60:274-83.

23. Pomerri F, Crimì F, Veronese N, Perin A, Lacognata C, Bergamo F, et al. Prediction of N0 irradiated rectal cancer comparing mri before and after preoperative chemoradiotherapy. Dis Colon Rectum 2017;60:1184-91.

24. Perez RO, Pereira DD, Proscurshim I, Gama-Rodrigues J, Rawet V, São Julião GP, et al. Lymph node size in rectal cancer following neoadjuvant chemoradiation--can we rely on radiologic nodal staging after chemoradiation? Dis Colon Rectum 2009;52:127884.

25. Heijnen LA, Maas M, Beets-Tan RG, Berkhof M, Lambregts DM, Nelemans PJ, et al. Nodal staging in rectal cancer: why is restag- ing after chemoradiation more accurate than primary nodal staging? Int J Colorectal Dis 2016;31:1157-62.

26. Beets-Tan RG, Beets GL, Borstlap AC, Oei TK, Teune TM, von Meyenfeldt MF, et al. Preoperative assessment of local tumor extent in advanced rectal cancer: CT or high-resolution MRI? Abdom Imaging 2000;25:533-41.

27. Maas M, Beets-Tan RG, Lambregts DM, Lammering G, Nelemans PJ, Engelen SM, et al. Wait-and-see policy for clinical complete responders after chemoradiation for rectal cancer. J Clin Oncol 2011;29:4633-40.

28. KSAR Study Group for Rectal Cancer. Essential items for structured reporting of rectal cancer MRI: 2016 Consensus Recommendation from the Korean Society of Abdominal Radiology. Korean J Radiol 2017;18:132-51.

29. Bhoday J, Smith F, Siddiqui MR, Balyasnikova S, Swift RI, Perez R, et al. Magnetic resonance tumor regression grade and residual mucosal abnormality as predictors for pathological complete response in rectal cancer postneoadjuvant chemoradiotherapy. Dis Colon Rectum 2016;59:925-33.

30. West MA, Dimitrov BD, Moyses HE, Kemp GJ, Loughney L, White $\mathrm{D}$, et al. Timing of surgery following neoadjuvant chemoradiotherapy in locally advanced rectal cancer - A comparison of magnetic resonance imaging at two time points and histopathological responses. Eur J Surg Oncol 2016;42:1350-8. 